\title{
Individualized eHealth Support for Oncological Therapy Management
}

\author{
Timo Schinköthe $e^{a, b}$ \\ a Breast Center, Department of Gynecology and Obstetrics and CCC Munich, University of Munich (LMU), \\ Munich, Germany; ${ }^{b}$ CANKADO Service $\mathrm{GmbH}$, Kirchheim b.München, Germany
}

Keywords

eHealth · Patient-reported outcome · Mobile medical device

\section{Abstract}

eHealth support for oncological therapy management has different faces. In addition to the direct support of therapy-supportive measures such as the promotion of physical activities, monitoring of the diet and observation of the quality of life through eHealth approaches are possible. Many solutions are now in transition between research and routine use. The most substantial evidence today is in the area of symptom monitoring. Independent studies have shown a significant survival benefit among other advantages as well. There is also a good overlap in eHealth and physical activity. More and more solutions are coming onto the market, which also has an impact for cancer patients. In the field of nutrition, there are only a few successful approaches both within and outside oncology. In contrast, the application of eHealth for the detection of disease-related malnutrition seems promising. Innovative approaches involving "artificial intelligence" or "Internet of Things" are slowly finding their place in both research and routine. For routine care, it will be necessary for doctors, nurses, and patients to know what a real medical eHealth application is and which are just lifestyle products without the needed quality for professional medical usage. @ 2019 S. Karger AG, Basel

\section{Introduction}

The term eHealth is not defined uniformly. In the beginning, eHealth meant the electronic storage of health or disease-related information. Today, however, eHealth is more about integrating Internet-based solutions into medical care and monitoring and improving fitness and health. In the era of the Nokia smartphones, one still distinguished "mobile Health" of eHealth applications. With the merging of mobile devices and conventional computers, this separation no longer makes sense today. Today, the term eHealth hides two worlds to be separated. In addition to lifestyle applications, this includes the field of medical devices.

As we move into eHealth therapy management today, we need to be aware that this is where legislation can come into play. When using drugs, it goes without saying that there are three phases of clinical trials followed by approval. In the field of eHealth, the legal provisions are little known. However, especially in oncological therapy management, we move very fast in the area of a medical device. Until the year 2010, the European Union always defined medical devices as objects. Software was only classified as a medical device, if the manufacturer intended it for use with a given medical device. The amendment of the Medical Device Directive 93/42/EEC in 2010 defined software as an independent medical device for the first time. Since then, software has been a medical device, if one of the descriptions in medical device definitions is fulfilled.

eHealth application in oncology is of particular importance when the software is intended for the "diagnosis, prevention, monitoring, treatment or alleviation of disease" [1] by the manufacturer. It is achieved very quickly in oncology when using an eHealth system; e.g., to record symptoms. If, for example, a breast cancer patient under lapatinib documents severe diarrhea, the intake should be discontinued according to the product information. eHealth complaint documentation systems for oncology

\section{KARGER}

(C) 2019 S. Karger AG, Base 
Fig. 1. Homepage of CANKADO (https:// www.cankado.com/).

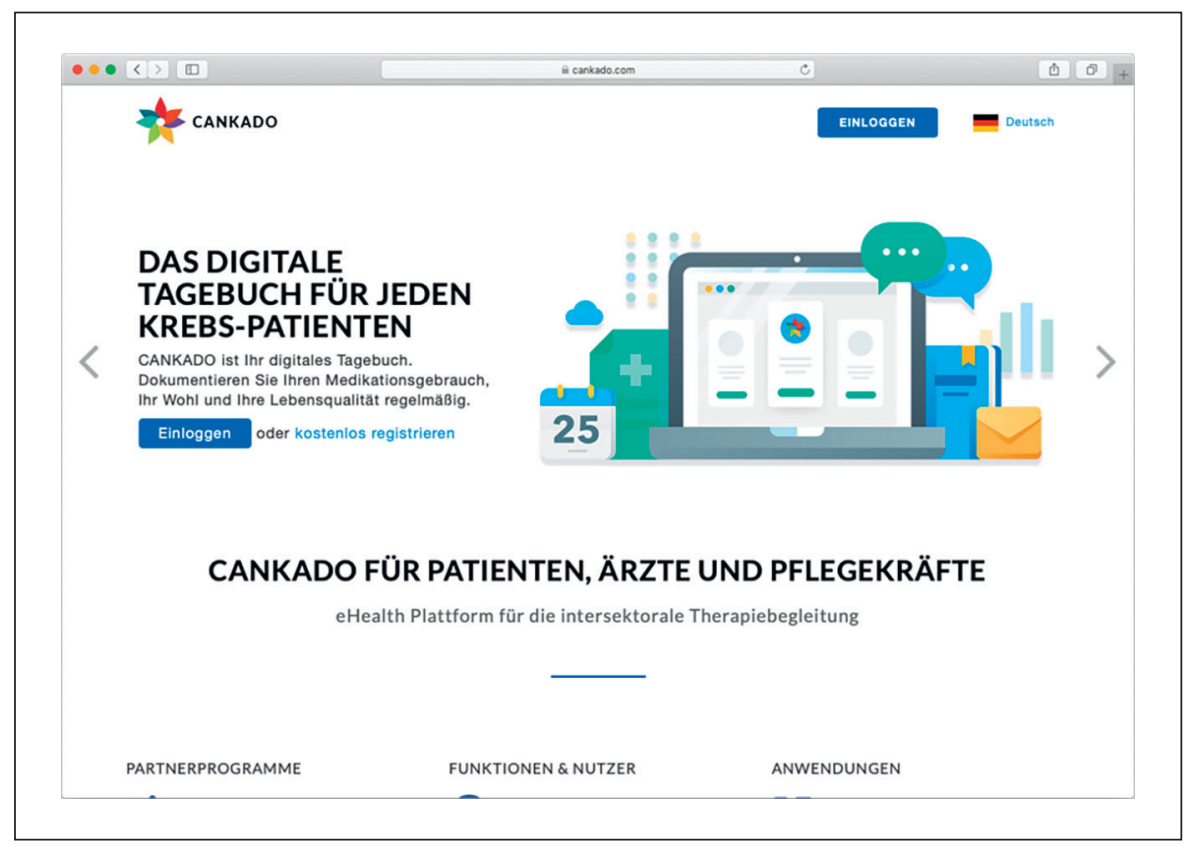

patients thus intervene in both surveillance and disease treatment and are therefore classified as medical devices.

In May 2020, a further tightening of the Medical Device Directive will come into force. Until now, all independent software systems have been classified as active medical devices of class I. These will be divided into risk categories next year. Depending on the type of application, software solutions are then subdivided from class I (no methodological risks) to class III (particularly high methodological risk). eHealth solutions to which the doctor is oriented in his therapy then at least belong to class IIa. Thus, by regulation, these solutions are equivalent to a diagnostic ultrasound device or an MRI [2]. In the USA, the regulations are a little bit easier. In 2015, the FDA provided a classification for Mobile Medical Devices, which says that software has to be compliant with the rules but does not need any registration or approval process in most of the oncological cases.

The vast majority of eHealth offerings are so-called lifestyle applications, which are not subject to regulation and can only rarely be used in the medical-therapeutic environment. In contrast to this are eHealth solutions, which are medical devices and can subsequently be used in professional care. Android and the Apple Store now have over two million apps available. Of the meanwhile around 200 billion app downloads per year, 10\% are in the area of lifestyle, health, and fitness. In contrast, there are currently only about 600 apps or software solutions registered as medical devices in Europe. This work should, therefore, give an overview of which studies are currently available in the field of oncological therapy management and how they should be evaluated concerning the possible use in routine care.

eHealth for Therapy Management

\section{eHealth in Therapy Management}

In therapy management, eHealth is mainly used in four areas. In addition to the monitoring of symptoms and side effects, these are psycho-oncology, nutrition, and physical activity.

\section{Monitoring of Symptoms}

eHealth received a lot of attention when the STAR Study (Symptom Tracking and Reporting for Patients) was presented at ASCO's 2017 Annual Meeting. Ethan Basch et al. [3, 4] demonstrated a 5-month survival advantage in outpatient chemotherapy for various solid tumors with an application of a simple digital questionnaire. In the STAR study, patients had to document symptoms once a week using a structured questionnaire, and if abnormalities were seen, these patients were contacted. In the CANKADO online system, this basic principle was adopted and adapted to the daily routine of care (Fig. 1). Changes in the general health status recognize the incidence of symptoms in CANKADO. Depending on this, situation-related symptom questionnaires are started. If symptoms are documented, the patient gets direct behavioral advice. Remote monitoring by a doctor or nurse is not necessary. CANKADO is a medical de vice [5].

Denis et al. [6,7] concentrated on advanced-stage lung cancer patients without evidence of disease progression after or during the initial treatment. Patients were randomly assigned in a multicenter phase III trial to compare a web-mediated follow-up algorithm (experimental arm), based on weekly self-scored patient symptoms with rou- 
Fig. 2. Homepage of Barbells for Boobs (https://www.barbellsforboobs.org/).

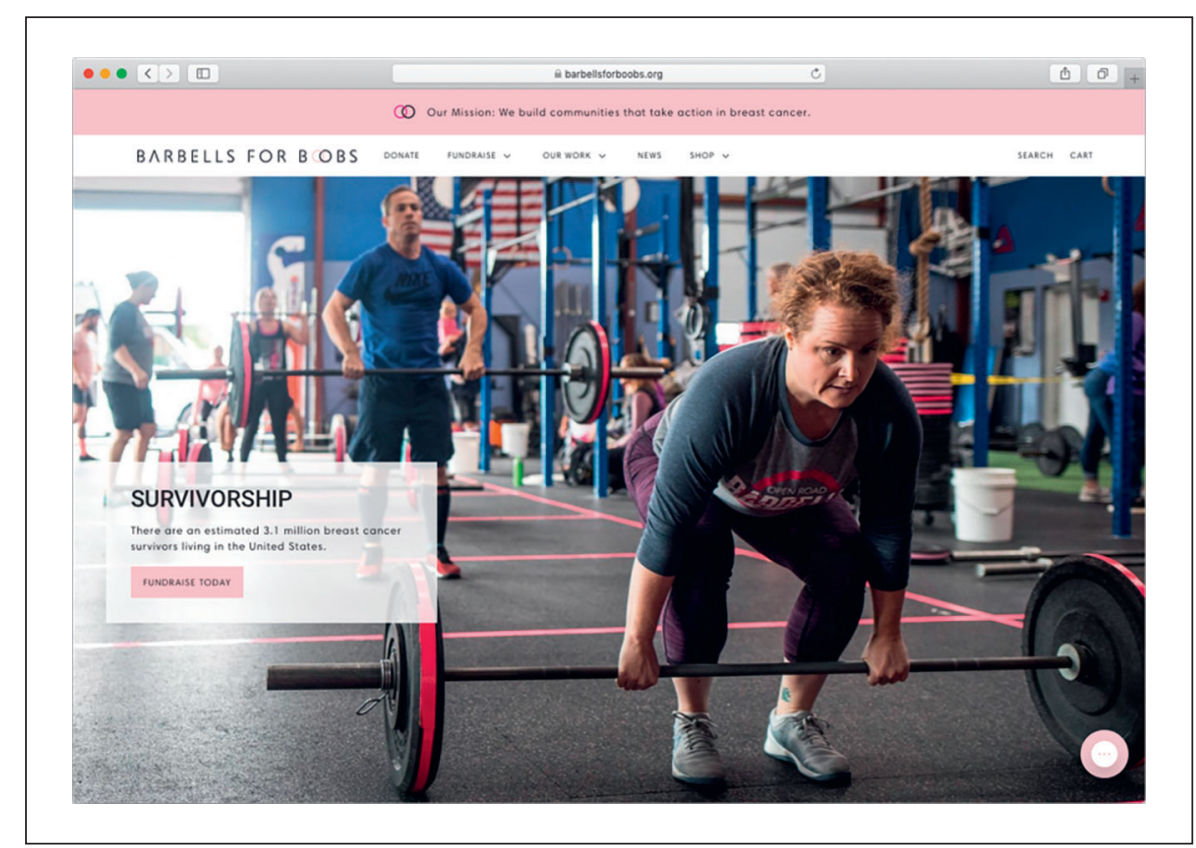

tine follow-up with CT scans scheduled every 3-6 months according to the disease stage (control arm). After 2 years of follow-up, 69 deaths were observed: 29 (47.5\%) in the intervention group and $40(66.7 \%)$ in the control group. The median overall survival was 22.5 months in the intervention group versus 14.9 months in the control group, without censoring for crossover (hazard ratio, 0.59 [95\% CI, 0.37-0.96]; $p=0.03)$. Denis et al. [6, 7] used MoovCare from Sivan Innovation, which is a medical device [8].

\section{Physical Activity}

A few years ago, a small, non-web-enabled pedometer came on the market. Today, these are also available in smart watches or smartphones. Almost all modern pedometers allow the movement information to be processed via an app.

The French ABLE study demonstrated a high willingness to participate in digitally controlled exercise training in patients with metastatic breast cancer $[9,10]$. Brigid Lynch's Australian group has been able to demonstrate that commercial consumer devices with the associated Fitbit, Jawebone, Garmin, or Polar apps can be used to increase physical activity in postmenopausal breast cancer patients [11].

In Spain, the online service e-CUIDATE (cuidate: Spanish for "take care of yourself") is available. e-CUIDATE offers patients an internet-based training plan tailored to their situation. This was evaluated in a two-arm study on breast cancer patients after adjuvant chemotherapy. The study showed both an improvement in the overall fitness and a significant reduction in fatigue [12].
In California, a few years ago, the "Barbells for Boobs" foundation was founded (Fig. 2). Barbells for Boobs' Resources after Diagnosis (RAD) online portal offers a training concept based on the CrossFit sport and specifically adapted to the needs of breast cancer patients. This is a so-called "Functional Fitness" sports program, which aims to increase everyday fitness [13].

The idea of combining physical activity improvement and social media is currently being investigated in China as part of a randomized study that is still ongoing. The participants in the intervention arm receive individually coordinated exercise instructions, which are recorded by a pedometer. Concomitantly, the participants are informed on the importance of exercise and rehabilitation via videos on social media [14].

\section{Psycho-Oncology}

A variety of apps are designed to capture the mood and quality of life of cancer patients. For the most part, these apps either combine advice on breast cancer or on specialized topics such as nutrition with the ability to document the self-perceived quality of life for themselves. All of these apps are in the lifestyle section. Exceptions of this are apps based on validated questionnaires such as the EQ-5D or the NCCN Distress Thermometer to perform psycho-oncological anamneses.

However, there are also eHealth applications that serve to improve the quality of life. One of the oldest projects is the Comprehensive Health Enhancement Support System (CHESS). CHESS started in 1987 as a local computer program at the University of Wisconsin-Madison and is now available online at chess.wisc.edu. In four studies in- 
volving approximately 1,400 breast cancer patients, the positive effect on the quality of life could be repeatedly demonstrated [15].

\section{Nutrition}

Concerning nutrition, there are currently two goals that are supported digitally. While on the one hand, there are cancer patients who want to lose weight, on the other hand, there are patients who suffer from disease-associated malnutrition.

A significant challenge to the wide-scale usage of eHealth interventions for weight-loss is that they typically suffer a steep decline in usage over the course of the intervention and have poor completion rates. Six different randomized trials looked at tailored eHealth weight loss support for healthy persons. After an observation period of 6 weeks to 12 months, 5 of the six studies reported a weight loss of between 0.5 and $2.8 \mathrm{~kg}$. Only one study was able to observe a weight reduction of up to $7.3 \mathrm{~kg}$ after 6 months. In addition, only one study had a duration of 24 months. After this time, the mean weight was $0.3 \mathrm{~kg}$ higher than at the time of starting [16].

There are currently only a few studies that have dealt with the topic of eHealth-supported weight loss in cancer patients. In five studies, a total of 211 survivors were accompanied by eHealth in different ways. The BMI ranged from 25 to 45 . The care time ranged from four weeks to 6 months. The interventions themselves were diverse. Two interventions used e-mailed lessons and feedback, one used tailored text messages, one provided participant input and advice via Facebook, and one delivered the intervention via phone, yet had subjects report dietary intake using text messages while weight and physical activity were obtained with wireless devices. Weight loss in these eHealth studies ranged from 0.2 to $2.3 \mathrm{~kg}$, which is in the range observed for healthy individuals [17].

An Austrian group investigated the possibility of detecting cancer-related malnutrition in outpatients using an app. Based on the Patient-Generated Subjective Global Assessment (PG-SGA), patients were followed in a 3-month pilot phase. Of the participating patients, $91 \%$ found the approach of Internet-based monitoring of cancer-related malnutrition to be meaningful [18]. Currently, a multinational study is underway to monitor patients using PG-SGA within CANKADO.

\section{Outlook}

Artificial intelligence has already reached pathology [19] and radiology [20] but failed in oncology treatment decisions [21]. However, it will be a matter of time when functioning applications will succeed in clinical trials.
Another emerging part in eHealth will be medical "Internet of Things" (IoT). IoT is the extension of Internet connectivity into physical devices and everyday objects. These devices can communicate and interact with others over the Internet and can be remotely monitored and controlled. Various studies have already tested the use of IoT for cancer detection. However, these approaches are currently not very promising [22]. The development status around small ECG devices such as the AliveCor by Kardia is quite different. Developed initially to detect cardiac arrhythmia [23], today these devices can be used in oncology in conjunction with the CANKADO app QTc Tracker for identifying drug-induced long QT syndrome.

\section{Conclusion}

In the field of eHealth research in oncological therapy management, there are a variety of movements. Clinicaltrials.gov currently has 41 active or non-recruiting eHealth trials in oncology. A central problem here, however, will be the contradiction between the high speed of software development on the one hand and the long duration of clinical trials on the other hand.

Clinical studies are excellent and essential but they were developed for static products, such as a drug. However, dynamic solutions, such as an eHealth platform, continue to evolve, even as a trial runs. This dynamic must be taken into account with new study concepts without undermining the quality and validity of clinical trials. Clinical trials are needed because the most important challenge in eHealth today is to distinguish serious apps from applications without evidence. For example, there are 340 breastfeeding apps out of which only 15 are evidence based [24]. The legislator has defined framework conditions for this. However, these must be made known and implemented accordingly. As long as physicians are unaware of these guidelines and no regulatory agencies are monitoring the implementation, it will be challenging to achieve eHealth quality standards and to maintain them nationwide. What is taken for granted in the field of medication should also be implemented in the area of eHealth. The more computer systems intervene in medical care, the more these should also follow comprehensible standards and be independently controlled.

Even more confusing is the situation when machine learning will find its way into medicine. This is mostly still in the area of visions of the future but is becoming increasingly real. It will be even harder to verify the evidence and make sure that the computer acts as we expect it to do. Last but not least, many people are still familiar with the previous year's results from IBM Watson. Inter- 
nal documents from IBM Watson Health indicate that the company's Watson for Oncology product often returns "multiple examples of unsafe and incorrect treatment recommendations" [21].

\section{Disclosure Statement}

The author is managing director and owner of CANKADO Service $\mathrm{GmbH}$.

\section{References}

1 COUNCIL DIRECTIVE 93/42/EEC of 14 June 1993 concerning medical devices: COUNCIL OF THE EUROPEAN COMMUNITIES; 2019 Available from: https://eur-lex.europa.eu/LexUriServ/LexUriServ.do?uri=CONSLEG: 1993L0042:20071011:en:PDF.

2 Giesselmann K. Medizinprodukte: risikoklasse für Apps steigt. Dtsch Arztebl. 2018; 115(12):A-538.

3 Basch E, Deal AM, Kris MG, Scher HI, Hudis CA, Sabbatini P, et al. Symptom Monitoring With Patient-Reported Outcomes During Routine Cancer Treatment: A Randomized Controlled Trial. J Clin Oncol. 2016 Feb; 34(6):557-65.

4 Basch E, Deal AM, Dueck AC, Scher HI, Kris MG, Hudis C, et al. Overall Survival Results of a Trial Assessing Patient-Reported Outcomes for Symptom Monitoring During Routine Cancer Treatment. JAMA. 2017 Jul;318(2): 197-8.

5 CANKADO. CANKADO; 2019. Available from: www.cankado.com.

6 Denis F, Lethrosne C, Pourel N, Molinier O, Pointreau Y, Domont J, et al. Randomized Trial Comparing a Web-Mediated Follow-up With Routine Surveillance in Lung Cancer Patients. J Natl Cancer Inst. 2017 Sep;109(9). https://doi.org/10.1093/jnci/djx029.

7 Denis F, Basch E, Septans AL, Bennouna J, Urban T, Dueck AC, et al. Two-Year Survival Comparing Web-Based Symptom Monitoring vs Routine Surveillance Following Treatment for Lung Cancer. JAMA. 2019 Jan; 321(3):306-7.

8 MoovCare. Sivan Innovation; [Available from: https://sivan-innovation.com/moovcare.

9 Delrieu L, Pérol O, Fervers B, Friedenreich C, Vallance J, Febvey-Combes O, et al. A Personalized Physical Activity Program With Activity Trackers and a Mobile Phone App for Patients With Metastatic Breast Cancer: Protocol for a Single-Arm Feasibility Trial. JMIR Res Protoc. 2018 Aug;7(8):e10487.
10 Fervers B, Delrieu L, Pérol O, Febvey-Combes O, Dufresne A, Bachelot T, et al. Poster display session: Breast cancer - early stage, locally advanced \& metastatic, CNS tumours, Developmental therapeutics, Genitourinary tumours - prostate \& non-prostate, Palliative care, Psycho-oncology, Public health policy, Sarcoma, Supportive care. ESMO 2018 Congress; suppl_8: Ann Oncol. 2018:viii90viii121.

11 Nguyen NH, Hadgraft NT, Moore MM, Rosenberg DE, Lynch C, Reeves MM, et al. A qualitative evaluation of breast cancer survivors' acceptance of and preferences for consumer wearable technology activity trackers. Support Care Cancer. 2017 Nov;25(11):337584.

12 Galiano-Castillo N, Cantarero-Villanueva I, Fernández-Lao C, Ariza-García A, Díaz-Rodríguez L, Del-Moral-Ávila R, et al. Telehealth system: A randomized controlled trial evaluating the impact of an internet-based exercise intervention on quality of life, pain, muscle strength, and fatigue in breast cancer survivors. Cancer. 2016 Oct;122(20):316674 .

13 Barbells for Boobs. 2019. Available from: https://www.barbellsforboobs.org.

14 Xiaosheng D, Xiangren Y, Shuyuan H, Dezong G, Mengyao C, Meng D. The effects of combined exercise intervention based on Internet and social media software for postoperative patients with breast cancer: study protocol for a randomized controlled trial. Trials. 2018 Sep;19(1):477.

15 Triberti S, Savioni L, Sebri V, Pravettoni G. eHealth for improving quality of life in breast cancer patients: A systematic review. Cancer Treat Rev. 2019 Mar;74:1-14.
16 Ryan K, Dockray S, Linehan C. A systematic review of tailored eHealth interventions for weight loss. Digit Health. 2019 Feb;5: 2055207619826685.

17 Harvey J, Dittus K, Mench E. eHealth and behavioral weight loss interventions for female cancer survivors: A review. Womens Health (Lond). 2017 Dec;13(3):80-8.

18 de Bruin JS, Schuh C, Seeling W, Luger E, Gall $M$, Hütterer E, et al. Assessing the feasibility of a mobile health-supported clinical decision support system for nutritional triage in oncology outpatients using Arden Syntax. Artif Intell Med. 2018 Nov;92:34-42.

19 Ehteshami Bejnordi B, Veta M, Johannes van Diest P, van Ginneken B, Karssemeijer N, Litjens $\mathrm{G}$, et al.; the CAMELYON16 Consortium. Diagnostic Assessment of Deep Learning Algorithms for Detection of Lymph Node Metastases in Women With Breast Cancer. JAMA. 2017 Dec;318(22):2199-210.

20 Houssami N, Kirkpatrick-Jones G, Noguchi $\mathrm{N}$, Lee CI. Artificial Intelligence (AI) for the early detection of breast cancer: a scoping review to assess AI's potential in breast screening practice. Expert Rev Med Devices. 2019 May;16(5):351-62.

21 Ross C. IBM's Watson supercomputer recommended 'unsafe and incorrect' cancer treatments, internal documents show. 25 July 2018; 2018. Available from: https://www.statnews.com/wp-content/uploads/2018/09/ IBMs-Watson-recommended-unsafe-andincorrect-cancer-treatments-STAT.pdf.

22 Ray PP, Dash D, De D. A Systematic Review of Wearable Systems for Cancer Detection: Current State and Challenges. J Med Syst. 2017 Oct;41(11): 180.

23 Garabelli P, Stavrakis S, Po S. Smartphonebased arrhythmia monitoring. Curr Opin Cardiol. 2017 Jan;32(1):53-7.

24 Sabesan S, Zalcberg J. Telehealth. N Engl J Med. 2018 Jan;378(4):402. 